\title{
A wavelet cluster-based band-pass filtering and envelope demodulation approach with application to fault diagnosis in a dry vacuum pump
}

\author{
W Jiang ${ }^{1,2}$, S K Spurgeon ${ }^{2}$, J A Twiddle ${ }^{2 *}$, F S Schlindwein ${ }^{2}$,Y Feng ${ }^{2}$, and S Thanagasundram ${ }^{2}$ \\ ${ }^{1}$ College of Mechanical Engineering, Yanshan University, Hebei, People's Republic of China \\ ${ }^{2}$ Department of Engineering, University of Leicester, Leicester, UK
}

The manuscript was received on 3 November 2006 and was accepted after revision for publication on 21 June 2007.

DOI: 10.1243/09544062JMES544

\begin{abstract}
A Morlet-like wavelet cluster-based method for band-pass filtering and envelope demodulation is described. Via appropriate choice of wavelet parameters, a wavelet cluster combined with multi-Morlet-like wavelets can be used as a band-pass filter with zero phase shift, flat topped pass-band and rapid attenuation in the transition band. It can be used to extract high frequency natural vibration components. The imaginary part of the Morlet-like wavelet cluster is the Hilbert transformation of its real part. This can be used to implement envelope demodulation and extract the envelope component of the high frequency resonance band. The method is applied for fault diagnosis relating to bearing defects in a dry vacuum pump. It is shown that the fault characteristic frequencies can be extracted effectively. The efficacy of the method is demonstrated in experimental studies.
\end{abstract}

Keywords: Morlet-like wavelet cluster, band-pass filtering, vibration analysis, envelope demodulation, fault diagnosis, dry vacuum pump, bearing fault

\section{INTRODUCTION}

When the working surface of a bearing component becomes damaged due to corrosive pitting, spalling, cracking or abrasion, for example, the damaged point will impact on other component surfaces during loaded motion and a periodical pulse force will be produced. This pulse force has a broad frequency band, which will include the high natural vibration frequency of the bearing system. Modulation of impact frequency to natural vibration frequency occurs.

Currently, envelope demodulation following bandpass filtering of the vibration signal is the most effective approach for diagnosis in bearings. By selecting a frequency band covering the high frequency resonance spectral peak, the fault characteristic frequencies can be identified. A band-pass filter is normally used in all such traditional envelope demodulation

*Corresponding author: Department of Engineering, University of Leicester, University Road, Leicester LE1 7RH, UK. email: jat7@le.ac.uk methods and careful filter design is important to avoid signal distortion [1].

Much work has been done in the spectrum analysis of vibration signals for monitoring rotating machinery such as rotary pumps, turbomolecular pumps, piston pumps, screw compressors, turbines, and motors, but very little has been done in the area of oil-free dry vacuum pumps [2]. However, the study of this kind of pump is important to industry because nowadays the dry vacuum pump is the common choice for clean room applications for integrated circuit semiconductor manufacturing. In metal etching and low-pressure chemical vapour deposition processes, chemical corrosive vapours are abundant. There are thus large amounts of residual particulates, and resultant pump failures must be avoided to prevent substantial financial losses due to the wafer costs involved. For these reasons, continuous real-time monitoring of dry vacuum pumps is desirable to reduce machine unscheduled downtime and maintenance costs.

In the current paper, a Morlet-like wavelet clusterbased method for band-pass filtering and envelope demodulation is proposed. By appropriate choice 
of wavelet parameters, the cluster combined with multi-Morlet-like wavelets can be used as a band-pass filter with zero phase shift, flat topped pass-band, and fast attenuation in the transition band. It can be used to extract high frequency natural vibration components. The imaginary part of the Morlet-like wavelet cluster is the Hilbert transformation of its real part. So the result of convolution of the sampled vibration signal with the wavelet cluster is the analytical signal. The square root of the quadratic sum of the real part and the imaginary part of the convolution result can realize the envelope demodulation.

The method is applied to fault diagnosis in a fivestage Roots and Claw mechanism dry vacuum pump. The fault characteristic frequencies are extracted effectively. The feasibility of the method is thus validated by experiment. In section 2 , the characteristics of the Morlet-like wavelet cluster are introduced. The envelope demodulation approach is described in section 3 . Section 4 presents the bearing fault detection problem and associated results.

\section{MORLET-LIKE WAVELET CLUSTER AND BAND-PASS FILTER}

\subsection{Single wavelet}

A single Morlet-like wavelet has the following mathematical representation

$$
\begin{aligned}
h(t) & =\frac{1}{\sqrt{2 \pi} a} \exp \left(-\frac{t^{2}}{2 a^{2}}\right) \exp \left(j 2 \pi f_{0} t\right) \\
& =\frac{1}{\sqrt{2 \pi} a} \exp \left(-\frac{t^{2}}{2 a^{2}}\right)\left[\cos \left(2 \pi f_{0} t\right)+j \sin \left(2 \pi f_{0} t\right)\right]
\end{aligned}
$$

with corresponding Fourier transformation

$$
H(f)=\exp \left(-\frac{a^{2}\left[2 \pi\left(f-f_{0}\right)\right]^{2}}{2}\right)
$$

where $a$ is a scalar parameter and $f_{0}$ is the central frequency. Equation (1) describes a single Morlet-like wavelet consisting of real part $h_{\mathrm{re}}(t)$ and imaginary part $h_{\mathrm{im}}(t)$. Equation (2) shows its spectrum is a bell-shaped Gaussian density function with central frequency $f_{0}$. By changing the parameter $a$, the height and width of the bell-shaped curve will be altered.

For specific parameters $a=0.2$ and $f_{0}=5$, the time domain waveform and spectrum curve are shown in Figs 1 and 2, respectively.

In fact, a single Morlet-like wavelet is a narrow bandpass filter with zero phase shift. It has high quality filtering characteristics. Through changing the parameter $a$, the bandwidth and attenuation of the transition zone can be altered. The filtered frequency band can be changed by adjusting the central frequency $f_{0}$.

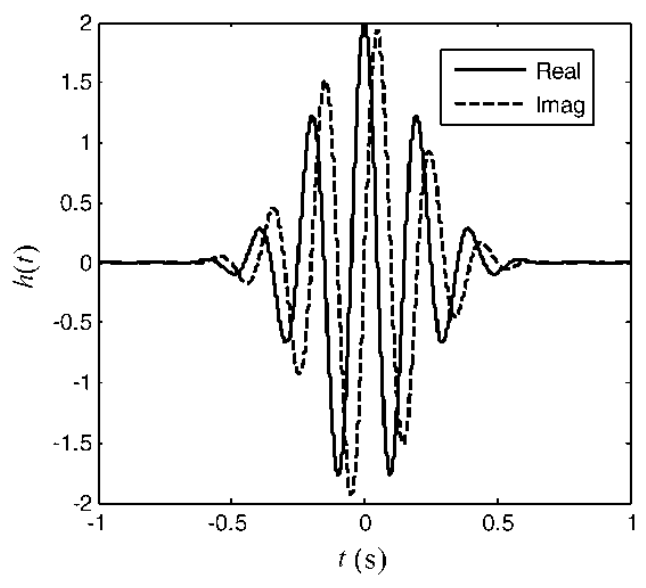

Fig. 1 Time domain waveform of a single Morlet-like wavelet

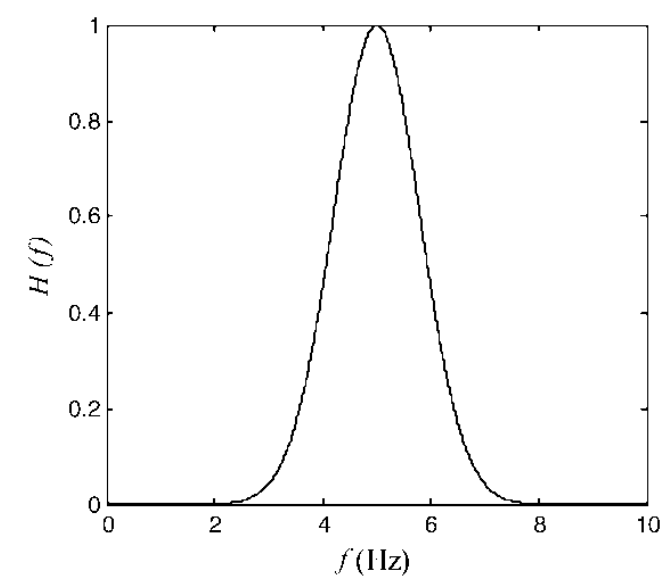

Fig. 2 Spectrum of a single Morlet-like wavelet

\subsection{Wavelet cluster}

A wavelet cluster is formed by combining $N+1$ single wavelets with differing central frequencies [3]. In this way a broad band-pass filter is constructed

$$
\begin{aligned}
h_{\mathrm{c}}(t)= & \frac{1}{\sqrt{2 \pi} a} \exp \left(-\frac{t^{2}}{2 a^{2}}\right)\left\{\exp \left[j 2 \pi f_{\mathrm{L}} t\right]\right. \\
& +\cdots+\exp \left[j 2 \pi\left(f_{\mathrm{L}}+k \Delta f\right) t\right] \\
& \left.+\cdots+\exp \left[j 2 \pi f_{\mathrm{H}} t\right]\right\} \\
k= & 0,1,2, \ldots, N, f_{\mathrm{H}}=f_{\mathrm{L}}+N \Delta f
\end{aligned}
$$

where $f_{\mathrm{L}}$ and $f_{\mathrm{H}}$ are, respectively, the lower and upper frequencies of the pass band, $\Delta f$ is the distance between the central frequencies of adjacent single wavelets. Through selecting suitable $a$ and $\Delta f$, a superposition spectrum providing good frequency characteristics such as flat pass-band and fast-attenuated transition band, can be obtained.

When $a=0.2, N=10, f_{\mathrm{L}}=10 \mathrm{~Hz}$, and $\Delta f=2 \mathrm{~Hz}$, then $f_{\mathrm{H}}=30 \mathrm{~Hz}$. The spectrum of the wavelet cluster is 
shown in Fig. 3. The cluster spectrum is not flat topped. When $a=0.2, N=10, f_{\mathrm{L}}=10 \mathrm{~Hz}$, and $\Delta f=1 \mathrm{~Hz}$, then $f_{\mathrm{H}}=20 \mathrm{~Hz}$, and the spectrum of the wavelet cluster is shown in Fig. 4. In this situation, the cluster has a flat-topped pass-band.

\subsection{Band-pass filter}

Convolution of the measured vibration signal with the wavelet cluster is given by

$$
s_{\mathrm{b}}(t)=s(t) \times h_{\mathrm{c}}(t)
$$

where $s_{\mathrm{b}}(t)$ is the component in the pass band $f_{\mathrm{L}} \sim f_{\mathrm{H}}$ of the original signal $s(t)$.

The use of the wavelet cluster as a band-pass filter has many merits. Its design and adjustment is convenient and straightforward. Depending on the actual requirements, selecting suitable $f_{\mathrm{L}}$ and $f_{\mathrm{H}}$, defines the frequency band to be analysed and, by adjusting $a$ and $\Delta f$, appropriate filter characteristics can

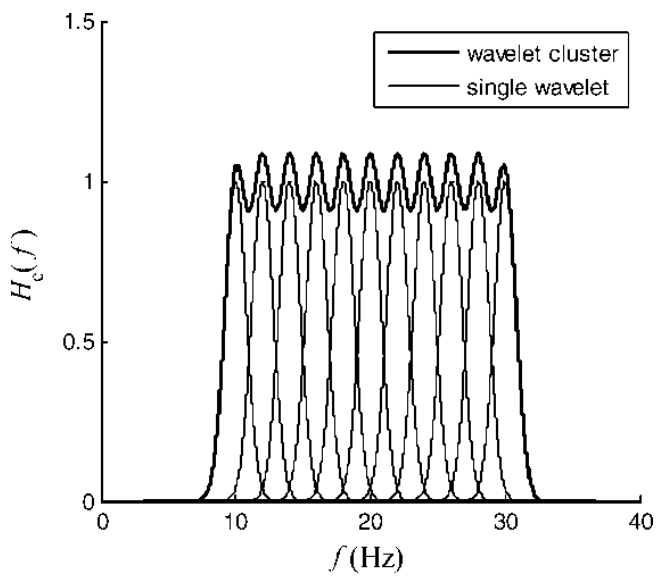

Fig. 3 Superposition spectrum (note that the pass band is not flat)

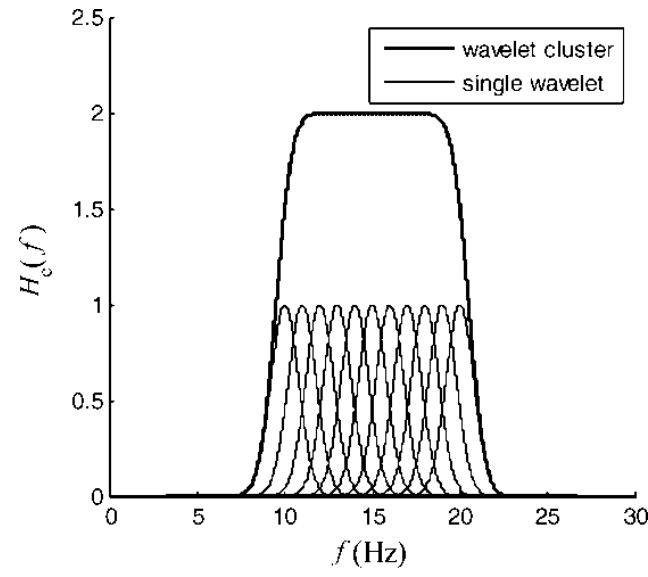

Fig.4 Superposition spectrum with flat-topped pass band be obtained. The carefully designed wavelet clusterbased band-pass filter has zero phase shift, a flat topped pass-band and fast attenuation in the transition band. The signal distortion due to non-linear phase of traditional infinite impulse response (IIR) filters can be avoided by using the wavelet cluster approach. Although zero phase shift can also be obtained using an finite impulse response (FIR) filter, narrow transition bands require a large number of coefficients for an FIR-based approach; furthermore, the use of the wavelet cluster as a digital filter produces the analytical signal and performs the envelope demodulation in one single step.

\section{ENVELOPE DEMODULATION}

\subsection{Hilbert transformation}

For a continuous time signal $x(t)$, its Hilbert transformation $\hat{x}(t)$ is defined as [4]

$$
\hat{x}(t)=x(t) \times \frac{1}{\pi t}
$$

$\hat{x}(t)$ can be thought of as the output of a filter having unit impulse response $h_{\mathrm{ht}}(t)=1 / \pi t$ and $x(t)$ is the input. The frequency response function of the Hilbert transformation is

$$
H_{\mathrm{ht}}(j f)=-j \operatorname{sign}(f)= \begin{cases}-j, & f>0 \\ j, & f<0\end{cases}
$$

Denoting $H_{\mathrm{ht}}(j f)=\left|H_{\mathrm{ht}}(j f)\right| e^{j \varphi_{\mathrm{ht}}(f)}$, then

$$
\begin{aligned}
& \left|H_{\mathrm{ht}}(j f)\right|=1 \\
& \varphi_{\mathrm{ht}}(f)= \begin{cases}-\pi / 2, & f>0 \\
\pi / 2, & f<0\end{cases}
\end{aligned}
$$

From the equations above, the Hilbert transformation is an all-pass filter with a frequency response characteristic of amplitude one. When a signal passes through the Hilbert transformation, its negative frequency components get a phase shift of $90^{\circ}$, and its positive frequency components get a phase shift of $-90^{\circ}$.

\subsection{Orthogonality of wavelet cluster}

According to equation (3), the real part of the wavelet cluster $h_{\mathrm{c}}(t)$ is

$$
\begin{aligned}
h_{\text {cre }}(t)= & \frac{1}{\sqrt{2 \pi} a} \exp \left(-\frac{t^{2}}{2 a^{2}}\right)\left[\cos \left(2 \pi f_{\mathrm{L}} t\right)\right. \\
& \left.+\cdots+\cos \left(2 \pi f_{k} t\right)+\cdots+\cos \left(2 \pi f_{\mathrm{H}} t\right)\right]
\end{aligned}
$$

where $f_{k}=f_{\mathrm{L}}+k \Delta f$. 
The corresponding Fourier transformation of $h_{\text {cre }}(t)$ is

$$
\begin{aligned}
H_{\text {cre }}(f)= & \frac{1}{2} \sum_{k=0}^{N}\left\{\exp -\left(\frac{a^{2}\left[2 \pi\left(f-f_{k}\right)\right]^{2}}{2}\right)\right. \\
& \left.+\exp \left(-\frac{a^{2}\left[2 \pi\left(f+f_{k}\right)\right]^{2}}{2}\right)\right\}
\end{aligned}
$$

The imaginary part of the wavelet cluster $h_{\mathrm{c}}(t)$ is

$$
\begin{aligned}
h_{\mathrm{cim}}(t)= & \frac{1}{\sqrt{2 \pi} a} \exp \left(-\frac{t^{2}}{2 a^{2}}\right)\left[\sin \left(2 \pi f_{\mathrm{L}} t\right)\right. \\
& \left.+\cdots+\sin \left(2 \pi f_{k} t\right)+\cdots+\sin \left(2 \pi f_{\mathrm{H}} t\right)\right]
\end{aligned}
$$

The corresponding Fourier transformation of $h_{\text {cim }}(t)$ is

$$
\begin{aligned}
H_{\text {cim }}(f)= & -\frac{j}{2} \sum_{k=0}^{N}\left\{\exp \left(-\frac{a^{2}\left[2 \pi\left(f-f_{k}\right)\right]^{2}}{2}\right)\right. \\
& \left.-\exp \left(-\frac{a^{2}\left[2 \pi\left(f+f_{k}\right)\right]^{2}}{2}\right)\right\}
\end{aligned}
$$

According to equations (6), (10), and (12), the Hilbert transformation of $h_{\text {cre }}(t)$ is

$$
\begin{aligned}
H_{\mathrm{cre}}(f) H_{\mathrm{ht}}(j f)= & -j \operatorname{sign}(f) H_{\mathrm{cre}}(f) \\
= & \frac{1}{2} \sum_{k=0}^{N}\left\{-j \exp \left(-\frac{a^{2}\left[2 \pi\left(f-f_{k}\right)\right]^{2}}{2}\right)\right. \\
& \left.+j \exp \left(-\frac{a^{2}\left[2 \pi\left(f+f_{k}\right)\right]^{2}}{2}\right)\right\} \\
= & H_{\text {cim }}(f)
\end{aligned}
$$

This means $h_{\text {cim }}(t)$ is the Hilbert transformation of $h_{\text {cre }}(t)$. Specifically $h_{\text {cre }}(t)$ and $h_{\text {cim }}(t)$ are mutually orthogonal.

From equation (4)

$$
\begin{aligned}
s_{\mathrm{b}}(t) & =s(t) \times h_{\mathrm{c}}(t)=s(t) \times h_{\mathrm{cre}}(t)+j s(t) \times h_{\mathrm{cim}}(t) \\
& =s_{\mathrm{bre}}(t)+j s_{\mathrm{bim}}(t)
\end{aligned}
$$

The Fourier transformation of equation (14) is

$$
\begin{aligned}
S_{\mathrm{b}}(f) & =S(f) H_{\mathrm{c}}(f)=S(f) H_{\mathrm{cre}}(f)+j S(f) H_{\mathrm{cim}}(f) \\
& =S_{\mathrm{bre}}(f)+j S_{\mathrm{bim}}(f)
\end{aligned}
$$

where

$$
\begin{aligned}
& S_{\text {bre }}(f)=S(f) H_{\text {cre }}(f) \\
& S_{\text {bim }}(f)=S(f) H_{\text {cim }}(f)
\end{aligned}
$$

Referring to equation (13), it can be obtained that

$$
S_{\mathrm{bim}}(f)=-j \operatorname{sign}(f) S_{\mathrm{bre}}(f)
$$

This means $s_{\text {bim }}(t)$ is the Hilbert transformation of $s_{\text {bre }}(t)$, so that $s_{\text {bre }}(t)$ and $s_{\text {bim }}(t)$ are mutually orthogonal.

It follows that the imaginary part of the convolution between the measured vibration signal and the wavelet cluster is the Hilbert transformation of its real part. Therefore, $s_{\mathrm{b}}(t)$ is the complex 'analytic signal'. The envelope component of the bandpass-filtered signal can be obtained by demodulation

$$
s_{\text {ben }}(t)=\sqrt{\left[s_{\text {bre }}(t)\right]^{2}+\left[s_{\text {bim }}(t)\right]^{2}}
$$

where $s_{\text {ben }}(t)$ is the envelope demodulated signal.

If faults are present the fault characteristic frequencies can be distinguished by analysing the power spectral density of $s_{\text {ben }}(t)$ [4].

According to the analysis above, the convolution calculation can realize the Hilbert transformation. Two processes of band-pass filtering and Hilbert transformation in the traditional envelope demodulation methods are combined in the convolution process in this approach.

\section{ENGINEERING APPLICATION}

\subsection{Dry vacuum pumps}

The aim of this study is to apply the wavelet clusterbased approach to diagnosis of a faulty bearing installed on a dry vacuum pump. The dry pump used in this experiment has a five-stage Roots and Claw design. The pump has one stage of two-lobed rotors near the high vacuum end and four stages of claws near the low vacuum end. The outlet port of the pump is located at the low vacuum end. Each rotor of the pump has a single row of deep groove ceramic bearings at both the low and high vacuum ends. The temperature of the pump is regulated by an external coolant water supply that prevents the pump from overheating. An inverter is used to control the speed of the pump and for this experiment the speed of the motor was fixed at $103 \mathrm{~Hz}$. A diagram of the dry vacuum pump is presented in Fig. 5 showing the vibration transducer mounting points close to the bearings at both the high vacuum end and the low vacuum end of the pump.

\subsection{Prediction of bearing defect frequencies}

Consideration of the bearing design, geometry, and rotational speed allows prediction of signature defect frequencies for a number of bearing faults, e.g. [5]. For the test pump used in the experiments the bearing parameters (nine balls, diameter $9.525 \mathrm{~mm}$, pitch diameter $46.2 \mathrm{~mm}$, and contact angle $24.97^{\circ}$ ) predict defect frequencies as follows. 


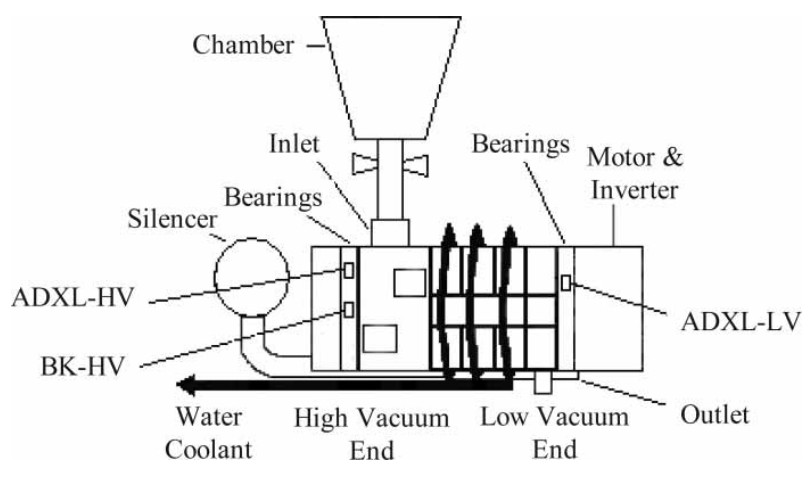

Fig. 5 Schematic configuration of dry vacuum pump

1. Inner race pass frequency $550 \mathrm{~Hz}$.

2. Single ball defect frequency $482 \mathrm{~Hz}$.

3. Outer race pass frequency $377 \mathrm{~Hz}$.

4. Cage defect frequency $42 \mathrm{~Hz}$.

\subsection{Transducers used to acquire vibration signals}

Acceleration signal was captured using ADXL105 and Brüel and Kjær 4370V accelerometers mounted radially on the dry vacuum pump near the high vacuum end. They are denoted in the results as ADXL-HV and BK-HV, respectively. Another ADXL105 accelerometer was mounted on the dry vacuum pump near the low vacuum end. It is denoted by ADXL-LV. The mounted positions of the transducers are shown in Fig. 5.

The ADXL105 accelerometer is a second-generation surface micro-machined device that carries a differential capacitive sensor and electronic signal conditioning circuitry on a single integrated chip [6]. The signals from the ADXL105 were filtered with a low pass anti-aliasing filter that was custom built in the laboratory. The filter has an eighth order elliptic lowpass characteristic with a cut-off frequency of $10 \mathrm{kHz}$. A relatively high order was implemented, as it was desired to have a steep cut-off rate at $10 \mathrm{kHz}$ and an attenuation of at least $70 \mathrm{~dB}$ in the stop band. Though the elliptic filter has a non-linear phase and hence a not-flat group delay, the phase characteristics of the signal are not important in this study and only the amplitude level of the vibration signal is of interest.

The signals from the Brüel and Kjær $4370 \mathrm{~V}$ accelerometer were conditioned using a Brüel and Kjær 2692 preamplifier which converted the signal from charge to voltage form, changed its gain and applied a band-pass filter from $0.1 \mathrm{~Hz}$ to $10 \mathrm{kHz}$ having $40 \mathrm{~dB} /$ decade attenuation slope at upper limiting frequency and lower limiting frequency.

The vibration signals were digitized using a 16-bit National Instruments NI6034E card at a sampling rate of $40 \mathrm{kHz}$. They were suitably amplified by the on-board gain amplifier on the ADC card before the A/D conversion took place to make full use of the available dynamic range.

\subsection{Constructing the wavelet cluster}

Figure 6 shows the power spectral density of sampled vibration signals from accelerometer BK-HV. A high frequency resonance peak appears at about $6 \mathrm{kHz}$. The desired pass-band of the filter is seen to be from 5 to $7 \mathrm{kHz}$.

The wavelet cluster-based flat-topping band-pass filter is constructed with $a=0.2, N=2000, f_{\mathrm{L}}=$ $5000 \mathrm{~Hz}, \Delta f=1 \mathrm{~Hz}$, and then $f_{\mathrm{H}}=7000 \mathrm{~Hz}$. The spectrum of the wavelet cluster is shown in Fig. 7. It has a steep cut-off frequency and flat-topped pass-band.

\subsection{Analysis process}

Block diagram of the wavelet cluster-based vibration signal-processing scheme is shown in Fig. 8.

The vibration signals are sampled at a rate of $40 \mathrm{kHz}$ from the accelerometers. The convolution between the original vibration signal and the wavelet cluster constructed in section 4.4 is performed. Through the convolution, the original vibration signal is both band-pass-filtered and the Hilbert transformation is implemented. Therefore, a complex analytical signal is attained with a frequency range of $5-7 \mathrm{kHz}$. The demodulation is realized by calculating the modulus of the complex analytical signal. The envelope signal is obtained by a tenth-order elliptical low-pass filter with a cut-off frequency of $1 \mathrm{kHz}$ and attenuation of $60 \mathrm{~dB}$ in the transition band. The data was then down-sampled to $2 \mathrm{kHz}$ as it was known that the frequencies of interest lie in the range of $0-1000 \mathrm{~Hz}$. After down-sampling the envelope signal, its mean value is removed by means of a sixth order high-pass elliptical filter with cut-off

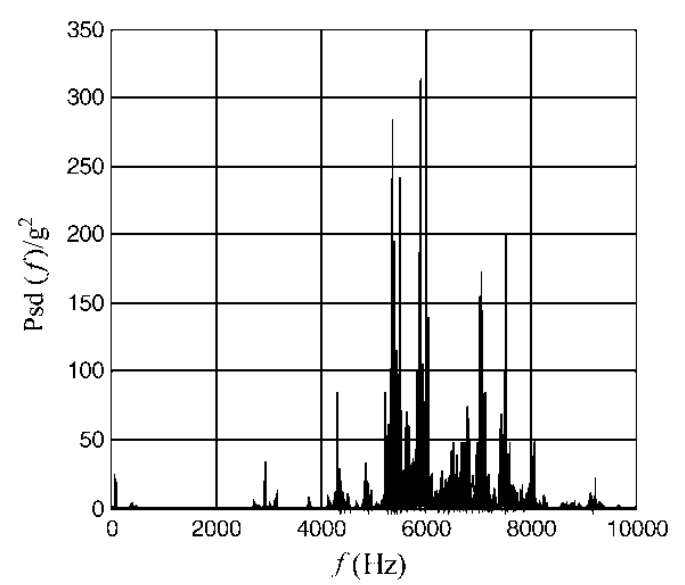

Fig. 6 Power spectral density of original vibration signal 


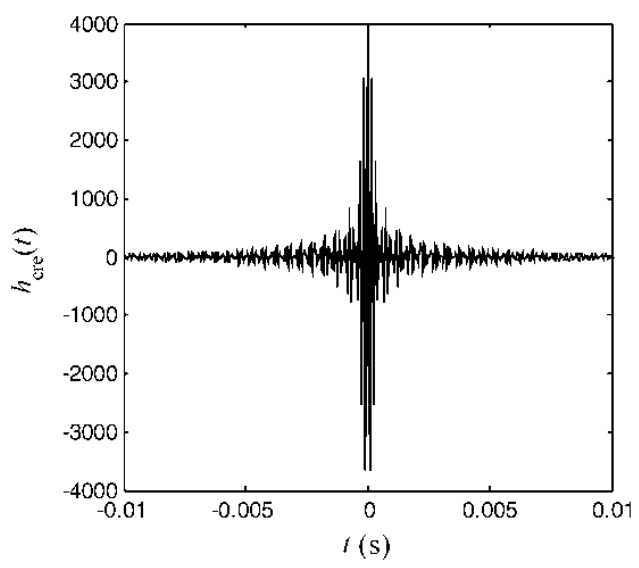

(a)

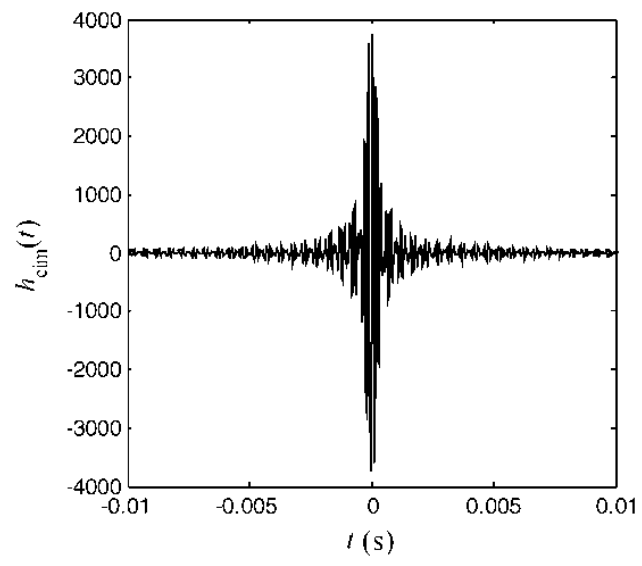

(b)

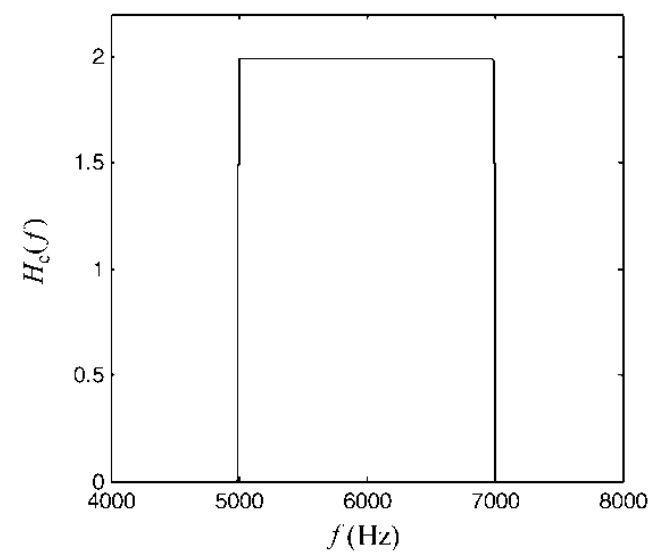

(c)

Fig. 7 Time waveform (a) and (b) and frequency spectrum (c) of wavelet cluster: (a) real part of wavelet cluster; (b) imaginary part of wavelet cluster; and (c) frequency spectrum of wavelet cluster

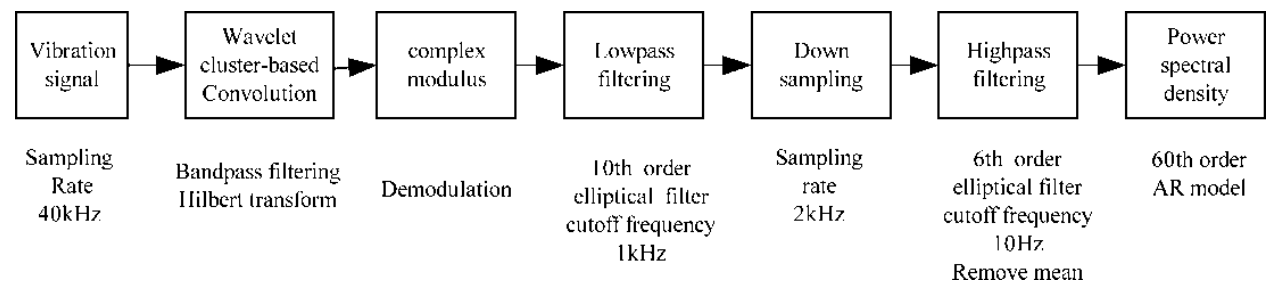

Fig. 8 Block diagram of wavelet cluster-based vibration signal processing

frequency of $20 \mathrm{~Hz}$ and attenuation of $60 \mathrm{~dB}$ in the transition band.

Finally, the power spectral density of the envelope signal is analysed by using an AR model. Previous studies [7] concluded that a model order for the pump is dependent on the number of samples per revolution. At 20 samples per revolution the minimum order is 20 and little or no performance improvements were seen for orders greater than 60 . A 60th order model is chosen here to provide sufficient resolution for the enveloped signal.

\subsection{Results and diagnosis}

Figures 9(a) to (c) show the results obtained via the processing methodology above for vibration signals from transducer ADXL-LV, ADXL-HV, and BK-HV, respectively. In these figures, the solid curves are the results with an inlet pressure $20 \mathrm{mbar}$ in a pump with faulty bearings. The dashed curves show the results with an inlet pressure of $90 \mathrm{mbar}$ in a pump with faulty bearings. The dotted curves show the results for a normal pump (under fault-free conditions) with an 


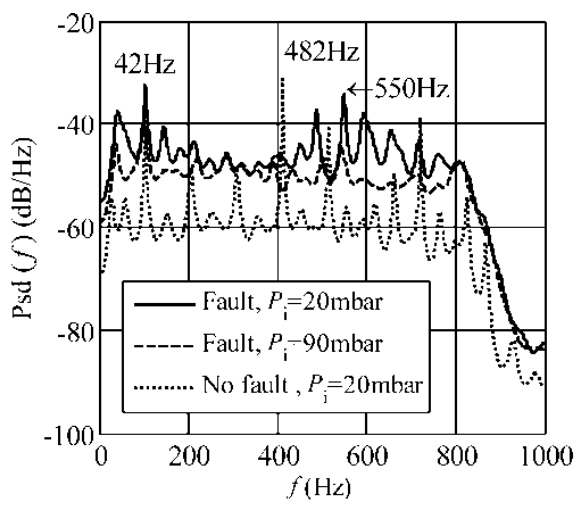

(a)

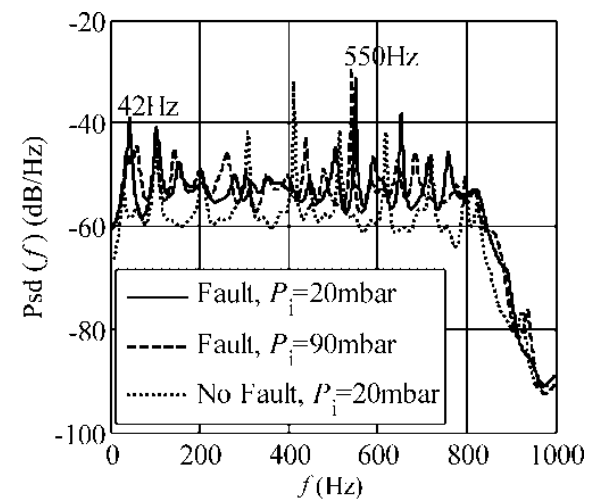

(b)

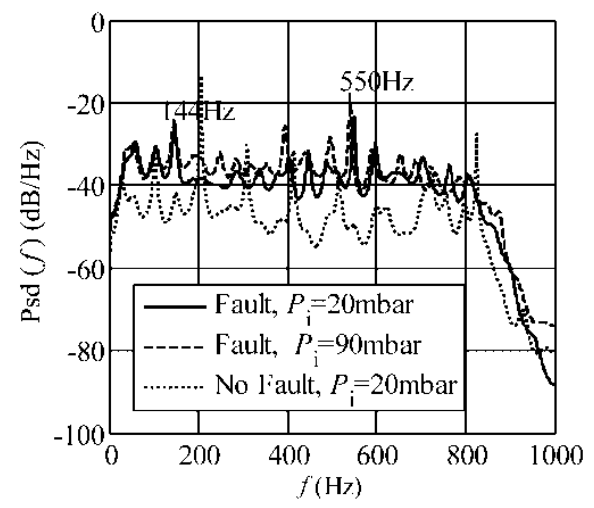

(c)

Fig. 9 Processing results for vibration signal from transducers ADXL-LV, ADXL-HV, and BK-HV (NB pump speed is $103 \mathrm{~Hz}$ at inlet pressure $20 \mathrm{mbar}$, and $101.5 \mathrm{~Hz}$ at $90 \mathrm{mbar}$ ). Power spectra from (a) ADXL-LV transducer signal; (b) ADXL-HV transducer signal, and BK-HV transducer signal

inlet pressure of 20 mbar. When calculating the power spectral density, 3072 data points were used.

In Fig. 9, the dotted curves show significant peaks in the fault-free spectra at the shaft rotating frequency $103 \mathrm{~Hz}$ and its higher-order harmonics.

The actual bearing fault condition analysed during the study was a group of indentations on the inner and outer race. A total of eight shallow indentations of approximately $1 \mathrm{~mm}^{2}$ (Fig. 10) were seeded around the rims of both the inner and outer races of the bearing. In practice such indentations could be caused by the ingress of soft foreign particles or pitting by aggressive media, for example refer [8]. According to section 4.2 these would cause signature frequency components at 550 and $377 \mathrm{~Hz}$, respectively.

Each of the fault data spectra in Figs 9(a) to (c) contains a significant peak at or close to $550 \mathrm{~Hz}$ confirming the presence of the fault condition. In addition to the $550 \mathrm{~Hz}$ peaks, Fig. 9(a), the ADXL-LV fault spectra, shows significant peaks close to $42 \mathrm{~Hz}$ (cage frequency), $482 \mathrm{~Hz}$ (ball pass frequency), and $550 \mathrm{~Hz}$ (inner race frequency). This suggests that if a fault occurs in one bearing component, mechanical
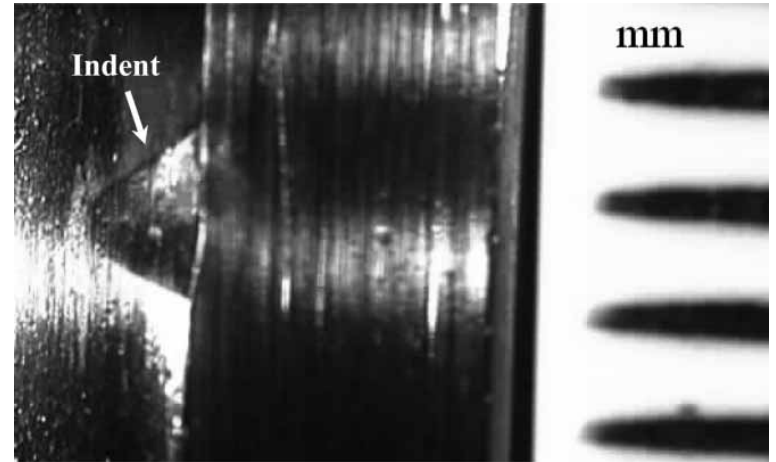

Fig. 10 An indentation in the rim of the bearing inner race

contact may cause vibration in other components. In Fig. 9(b), the ADXL-HV fault spectra also show significant peaks close to $42 \mathrm{~Hz}$ (cage frequency), and in Fig. 9 (c) a peak at approximately $144 \mathrm{~Hz}$ is visible which is not explained by a known defect frequency.

The fault detection scheme is sensitive to even the small indentations tested here that represent a low 
level of damage to the bearing as pump performance was not affected and there were no other apparent symptoms such as excess noise audible to the operator.

Fault symptoms generated by a HV-end bearing defect are transmitted well by the metal body of the pump so that fault detection is not sensitive to the location of the transducer and signals from all three transducers clearly indicate the presence of a fault. However, further work is required to determine whether it is possible to identify which bearing is carrying the fault condition.

\section{CONCLUSIONS}

A single Morlet-like wavelet can be used as a narrow band-pass filter. A Morlet-like wavelet cluster convolution can achieve band-pass filtering and Hilbert transformation simultaneously. The analysis process involved in traditional envelope demodulation is simplified.

The wavelet cluster-based band-pass filter has a zero phase-shift characteristic, avoiding the phase distortion of traditional filters. Through selecting suitable $a$ and $\Delta f$, a superposition spectrum with the required frequency characteristics of a flat pass band and rapidly attenuated transition band, can be obtained.

The wavelet cluster-based filtering and demodulation approach can be applied for fault diagnosis in a dry vacuum pump. Experimental results show the effectiveness of the method in fault diagnosis.

\section{ACKNOWLEDGEMENTS}

This work is supported financially by the Doctoral Fund of China Hebei Province under project number B2004128 and EPSRC grant GR/S42866/01. The authors would also like to thank BOC Edwards for the supply of the iGX dry vacuum pump and Barden Bearings for providing the ceramic ball bearings for the experimental investigations. This support is gratefully acknowledged. Also, thanks to Simon Lawes in the Materials Laboratory, Department of Engineering, University of Leicester for the photography included in the current paper.

\section{REFERENCES}

1 Jiancang, M. and Lei, L. Wavelet transform-based envelope analysis and its application in fault diagnosis. Mech. Sci. Technol., 1996, 15(3), 585-588.

2 Sabin, E.Vibration analysis of dry pumps. Semicond. Int., 1995, 18(8), 249-250.

3 Jiancang, M., Qibin, W., Jianwu, W., and Lei, L. Spectrum zoom analysis method based on wavelet transform. Signal Process., 1997, 13(3), 274-279.
4 Xiaojian, M., Ruiqi, C., and Wenying, W. The comparison and application of envelope demodulation in machine fault diagnosis. J. Donghua Univ., 2001, 27(5), 105-108.

5 Rubini, R. and Meneghetti, U. Application of the envelope and wavelet transform analyses for the diagnosis of incipient faults in ball bearings. Mech. Syst. Signal Process., 2001, 15(2), 287-302.

6 Thanagasundram, S. and Schlindwein, F. S. Comparison of integrated micro-electrical-mechanical system and piezoelectric accelerometers for machine condition monitoring. Proc. IMechE, Part C:J. Mechanical Engineering Science, 2005, 220(C8), 1135-1146.

7 Thanagasundram, S. and Schlindwein, F. S. Autoregressive order selection for rotating machinery. Int. J. Acoust. Vibr., 2005, 11(3), 144-154.

8 FAG Bearings Corporation. Rolling bearing damage recognition of damage and bearing inspection, publication number WL 82 102/2, 2003. FAG Kugelfischer AG, Business Unit Industrial, Bearings and Services, Postfach 1260 D-97419 Schweinfurt, Georg-Schäfer-Strasse 30, 97421 Schweinfurt.

\section{APPENDIX}

\section{Notation}

$f_{\mathrm{H}}$

$f_{\mathrm{L}}$

$h_{\mathrm{c}}(t)$

$h_{\text {cim }}(t)$

$h_{\text {cre }}(t)$

$h_{\mathrm{ht}}(t)$

$H(f)$

$H(t)$

$H_{\text {cim }}(f)$

$H_{\text {cre }}(f)$

$H_{\mathrm{ht}}(j f)$

$\left|H_{\mathrm{ht}}(j f)\right|$

$P_{\mathrm{i}}$

$s(t)$

$s_{\mathrm{b}}(t)$

$s_{\text {ben }}(t)$

$s_{\text {bim }}(t)$

$s_{\text {bre }}(t)$

$S_{\text {bim }}(f)$

$S_{\text {bre }}(f)$

$\hat{x}(t)$

$\Delta f$

upper frequency of pass-band of filter $h_{\mathrm{c}}(t)$

lower frequency of pass-band of filter $h_{\mathrm{c}}(t)$

Morlet-like wavelet cluster

imaginary part of $h_{\mathrm{c}}(t)$

real part of $h_{\mathrm{c}}(t)$

unit impulse response function of Hilbert transformer

frequency spectrum of $h(t)$

single Morlet-like wavelet function

frequency spectrum of $h_{\text {cim }}(t)$

frequency spectrum of $h_{\text {cre }}(t)$

frequency response function of Hilbert transformer

amplitude frequency response characteristics of Hilbert transformer

pump inlet pressure (mbar)

original sampled vibration signal

component in the pass band $f_{\mathrm{L}} \sim f_{\mathrm{H}}$ of the original signal $s(t)$

envelope demodulated signal

imaginary part of $s_{\mathrm{b}}(t)$

real part of $s_{\mathrm{b}}(t)$

frequency spectrum of $s_{\mathrm{bim}}(t)$

frequency spectrum of $s_{\text {bre }}(t)$

Hilbert transformation of the continuous time signal $x(t)$

distance between central frequencies of adjacent single wavelets in wavelet cluster

$\varphi(f) \quad$ phase frequency response characteristics of Hilbert transformer 